https://helda.helsinki.fi

\title{
Hemodialysis in children with ventriculoperitoneal shunts: prevalence, management and outcomes
}

\section{Wright, Elizabeth}

2016-01-01

Wright, E , Fischbach, M , Zaloszyc, A, Paglialonga , F , Aufricht, C , Dufek, S, Bakkaloglu , S, Klaus , G , Zurowska , A, Ekim , M , Ariceta , G , Hölttä , T , Jankauskiene , A , Schmitt , C P , Stefanidis , C J , Walle , J V , Vondrak, K, Edefonti , A, Shroff , R \& Working , E P D 2016 , ' Hemodialysis in children with ventriculoperitoneal shunts: prevalence, management and outcomes ', Pediatric Nephrology, vol. 31 , no. 1 , pp. 137-143 . https://doi.org/10.1007/s00467-015-3204-5

http://hdl.handle.net/10138/159063

https://doi.org/10.1007/s00467-015-3204-5

publishedVersion

Downloaded from Helda, University of Helsinki institutional repository.

This is an electronic reprint of the original article.

This reprint may differ from the original in pagination and typographic detail.

Please cite the original version. 


\title{
Hemodialysis in children with ventriculoperitoneal shunts: prevalence, management and outcomes
}

\author{
Elizabeth Wright $^{1} \cdot$ Michel Fischbach $^{2} \cdot$ Ariane Zaloszyc $^{2} \cdot$ Fabio Paglialonga $^{3}$. \\ Christoph Aufricht $^{4}$ - Stephanie Dufek ${ }^{1}$ - Sevcan Bakkaloğlu ${ }^{5}$ - Günter Klaus ${ }^{6}$. \\ Aleksandra Zurowska ${ }^{7}$ - Mesiha Ekim ${ }^{8}$. Gema Ariceta ${ }^{9}$. Tuula Holtta ${ }^{10}$. \\ Augustina Jankauskiene ${ }^{11}$. Claus Peter Schmitt ${ }^{12}$. Constantinos J. Stefanidis ${ }^{13}$. \\ Johan Vande Walle ${ }^{14} \cdot$ Karel Vondrak $^{15}$ - Alberto Edefonti $^{3} \cdot$ Rukshana Shroff $^{1}$ • on \\ behalf of the European Paediatric Dialysis Working Group
}

Received: 23 April 2015 /Revised: 13 August 2015 / Accepted: 28 August 2015 /Published online: 19 September 2015 (C) IPNA 2015

\begin{abstract}
Background Hemodialysis (HD) in children with a concomitant ventriculoperitoneal shunt (VPS) is rare. Registry data suggest that peritoneal dialysis with a VPS is safe, but little is known about HD in the presence of a VPS.

Methods We performed a 10-year survey to determine the prevalence of a VPS, complications and outcome in children with a VPS on HD in 15 dialysis units from the 13 countries participating in the European Pediatric Dialysis Working Group.

Results Eleven cases of HD with a VPS were reported (prevalence $1.33 \%$; 328 patient-months) and compared with prospective Registry data. The median age at start of dialysis was 9.6 [inter-quartile range (IQR) 1.0-15.0] years and median HD vintage was 2.4 (IQR 1.7-3.0) years. Dialysis was performed through a central venous line (CVL) and through an arteriovenous fistula in six and five children, respectively. Three CVL infections occurred in two children, but these children did not develop VPS infections or meningitis. Symptoms
\end{abstract}

Rukshana Shroff

Rukshana.Shroff@gosh.nhs.uk

1 Nephro-Urology Unit, Great Ormond Street Hospital for Children, NHS Foundation Trust, London WC1N 3JH, UK

2 Hopital de Hautepierre, Strasbourg, France

3 Fondazione IRCCS Ca' Grande Ospedale Maggiore Policlinico, Milan, Italy

4 University Hospital of Vienna, Vienna, Austria

5 Ankara University Hospital, Ankara, Turkey

6 KfH Pediatric Kidney Center, Marburg, Germany of hemodynamic instability were reported in six (55\%) children at least once per week, with hypotension or hypertension occurring in four of these children and nausea, vomiting and headaches occurring in two; four other children reported less frequent symptoms. Seizures on dialysis occurred in two children, at a frequency of less than once per month, with one child also experiencing visual disturbances. During followup (median 4.0; IQR 0.38-7.63 years), three children remained on HD and eight had a functioning transplant. No patients were switched to PD.

Conclusions Hemodialysis in children with a VPS is safe, but associated with frequent symptoms of hemodynamic instability. No episodes of VPS infection or meningitis were seen among the children in the survey, not even in those with CVL sepsis.

Keywords Hemodialysis · Ventriculoperitoneal shunt · Children $\cdot$ Central venous line $\cdot$ Seizures

\footnotetext{
Gdansk University Medical School, Gdansk, Poland

Gazi University Hospital, Ankara, Turkey

9 University Hospital Vall d'Hebron, Barcelona, Spain

10 University of Helsinki, Helsinki, Finland

11 Vilnius University, Vilnius, Lithuania

12 Center for Pediatric \& Adolescent Medicine, Heidelberg, Germany

13 “A \& P Kyriakou”, Children's Hospital, Athens, Greece

14 Universitair ziekenhuis Gent, Ghent, Belgium

15 University Hospital Motol, Prague, Czech Republic
} 


\section{Introduction}

Dialysis in children with concomitant ventriculoperitoneal shunts (VPS) is uncommon, with only anecdotal reports in the literature, both in adults [1] and children [2,3]. The potential for developing shunt infections is increased in children on hemodialysis (HD), who also show a poor tolerance of dialysis due to the possibility of fluid shifts induced during a HD session leading to abrupt changes in the intracerebral pressure. There is a single case report of an adult on HD who developed VPS-associated sepsis [4]. Similarly, children with VPS on peritoneal dialysis (PD) are thought to be at risk of shunt infection, and the presence of a 'foreign body' in the peritoneal cavity is considered to be a contraindication for PD by many nephrologists. However, a recent survey from the International Pediatric Peritoneal Dialysis Network (IPPDN) found no evidence to support an increased risk of peritonitis or VPS infection in children on PD [5].

We conducted a 10-year survey across 15 pediatric dialysis centers from the 13 European Union countries that represent the European Pediatric Dialysis Working Group (EPDWG) to review the prevalence, safety, complications and outcome of HD in children with VPS.

\section{Subjects and methods}

All children with VPS receiving HD or hemodiafiltration (HDF) in 15 European pediatric nephrology centers between April 2004 and March 2014 were included in the survey. Patients were identified from recall and checked against the dialysis database maintained in each unit. Anonymized retrospective data collection was performed via questionnaires distributed to all 15 centers represented in the EPDWG. Information on the demographics of the chronic HD population in each center was collected, together with additional detailed information on patient characteristics, specifics of the HD therapy, management of complications and outcome for each child with a VPS on HD. Hemodynamic instability was defined for this survey as hypotension, hypertension, nausea, vomiting, headaches, visual disturbances or seizures. Data were submitted to a central unit for analysis (Great Ormond Street Hospital, London) and verified by email correspondence and at meetings of the group. Ethical approval for retrospective case-note review was obtained as per local requirements. For comparison, episodes of symptomatic hemodynamic instability were recorded in a cohort of children on HD who did not have VPS matched for age, gender and dialysis vintage. Data from children dialyzed contemporaneously to our study cohort were not available, so we collected details from the International Pediatric Hemodialysis Network (IPHN; http://www.pedpd.org/), an international registry of pediatric HD patients that maintains a prospective database on dialysis technique, outcomes and management.

\section{Shunt characteristics}

All shunts were inserted prior to the commencement of dialysis. Shunt position was ventriculoperitoneal in ten children and ventriculoatrial (VA) in one child. One VPS was later changed to a VA shunt 6 months after the commencement of HDF due to malfunction. The shunts were inserted at a median age of 6 months [interquartile range (IQR) 1 day-12 months]. Five children (45\%) had their shunts revised 11 times in total. On ten occasions this was for shunt malfunction and took place before dialysis was commenced. One child had the shunt externalized as a precaution following a renal transplantation that was complicated by acute appendicitis and candida wound infection with graft failure and a return to HD within 8 days.

\section{Dialysis prescriptions}

Nine children were on HD as the predominant dialysis modality and two were on HDF. It was not possible to compare modalities due to the small numbers of patients. All HD sessions were performed three times a week, for $4 \mathrm{~h}$ per session. Two children were started on HDF from the outset, with three to five sessions per week, for $3-4 \mathrm{~h}$ per session. The dialysis modality and duration followed unit policy and was not changed in those with a VPS.

Dialysate prescriptions varied between centers. The median dialysate sodium and bicarbonate level was 140 (IQR 138143) and 34 (IQR 32-35) mMol/l, respectively. The median dialysate temperature was 36.5 (IQR $36-37$ ) ${ }^{\circ} \mathrm{C}$. All but one center used a glucose-containing dialysate. Sodium and ultrafiltration profiling was routinely performed in two centers (1 patient each).

\section{Statistical analysis}

Since most data were non-parametric, the results are presented as the median and IQR. Due to the small number of patients only descriptive statistics were possible.

\section{Results}

During the study period there were 11 children with VPS on HD distributed across eight of the 15 centers representing the EPDWG. Three centers were treating two children each and five centers were treating one child each. During the 10-year study period 824 children received HD. The prevalence of HD patients with a VPS was $1.33 \%$, with 322 patient-months experience of HD in children with a VPS. 
Patient characteristics are presented in Table 1. The median age at start of dialysis was 9.6 (IQR 6-13) years. The median time on dialysis with a VPS was 2.4 (IQR 1.7-3.0) years. Seven $(64 \%)$ patients had residual renal function when HD was first started; of these, one subsequently became anuric and one with focal segmental glomerulosclerosis had bilateral nephrectomies. The two children with VA shunts both had an arteriovenous fistula (AVF), formed in the non-dominant hand, which was also contralateral to the VA shunt. All central venous lines (CVLs) were in the internal jugular or brachiocephalic veins. All access was achieved using the double-lumen or double-needle technique. No child had ever received $\mathrm{PD}$, and no patient had switched to $\mathrm{PD}$ as a result of symptoms.

\section{Complications of dialysis}

Infections Three CVL infections were reported in two children (18\%). The causal organisms were methicillin-resistant Staphylococcus aureus, Staphylococcus haemolyticus and Streptococcus in one case each. None of the CVL infections led to VPS infection or meningitis. There were no cases of primary VPS infection or meningitis in any child once established on HD. One child had the VPS temporarily externalized as a precautionary measure to minimize the risk of an ascending Candida infection following a traumatic renal transplantation complicated by appendicitis and an infected surgical wound; no infection occurred.

Hemodynamic complications Of the 11 children included in the survey, six $(55 \%)$ had symptoms of hemodynamic instability as described in detail in Table 1 . Five of the six children with frequent symptoms had a residual urine output, but one child continued to experience symptoms following bilateral nephrectomies. The three children who were anuric experienced minimal or no symptoms. In a limited number of patients there was no correlation between the ultrafiltration volume per session and hemodynamic complications, but a complete set of data was not available for all patients. The two children who were started on HDF from the outset (and were also anuric) had the fewest symptoms. The frequency of symptoms is described in Table 2.

Seizures and visual disturbances Two children (18\%) on dialysis experienced seizures, both of whom had been diagnosed with epilepsy prior to commencement of dialysis. Both had seizures at a frequency of less than once per month. One child, who had an underlying diagnosis of hydrocephalus and focal segmental glomerulosclerosis but no other abnormalities on cranial computed tomography imaging, was known to have tonic/clonic seizures of short duration that were triggered by intercurrent illness even prior to the commencement of dialysis and was treated with carbamazepine. Two seizures occurred in this child during HD, both lasting $<5 \mathrm{~min}$ and resolving spontaneously. The carbamazepine dose was increased, and no further seizures were noted. This child also had occasional visual disturbances that were associated with intradialytic hypotension that occurred infrequently (less than once a month).

The second child, who had meningomyelocele but no other abnormalities on cranial magnetic resonance imaging scan, had short-lasting and self-resolving tonic-clonic seizures on dialysis that were thought to be triggered by fluid shifts during dialysis. No response was seen with mannitol or anti-epileptic treatment. In both cases the seizures were not attributed to shunt dysfunction or shunt infection.

For comparison, data from IPHN showed that four of 43 (9\%) children dialyzed in our centers developed infrequent complications of nausea or headaches and that two others $(4.6 \%)$ had frequent headaches over a median study duration of 6 months.

\section{Management of dialysis complications}

Three children $(27 \%)$ were switched from HD to HDF in an attempt to achieve better control of their fluid status. This change was reported to have reduced the frequency of intradialytic hypotensive episodes in two of the children. For the two children who demonstrated frequent intradialytic complications (nausea, vomiting and headache at least once a week), the dialysis session was increased from 4 to $5 \mathrm{~h}$ in one child and to up to $8 \mathrm{~h}$ in the other child; however, in both cases the symptoms continued to occur. The dialysate temperature was reduced in two children. Dialysate sodium was altered in three cases, with sodium profiling in one case, but this was abandoned as it proved to be ineffective. No child was switched to daily dialysis sessions.

Intradialytic complications were most commonly managed by adjusting the ultrafiltration rate as the first step: ultrafiltration was temporarily stopped in eight (73 \%) children, with three $(27 \%)$ children requiring a decrease in the total ultrafiltration volume and seven children (64\%) requiring the administration of a saline bolus. Three $(27 \%)$ children frequently required early discontinuation of the dialysis session. One child required prophylactic mannitol during each dialysis session, and in another child mannitol was used when symptoms of hypotension developed.

\section{Outcome}

At final follow-up after a median of 4.0 (IQR 0.38-7.63) years, eight children have a functioning transplant and three remain on HD. No child converted from HD to PD during the study period. There were no deaths. 


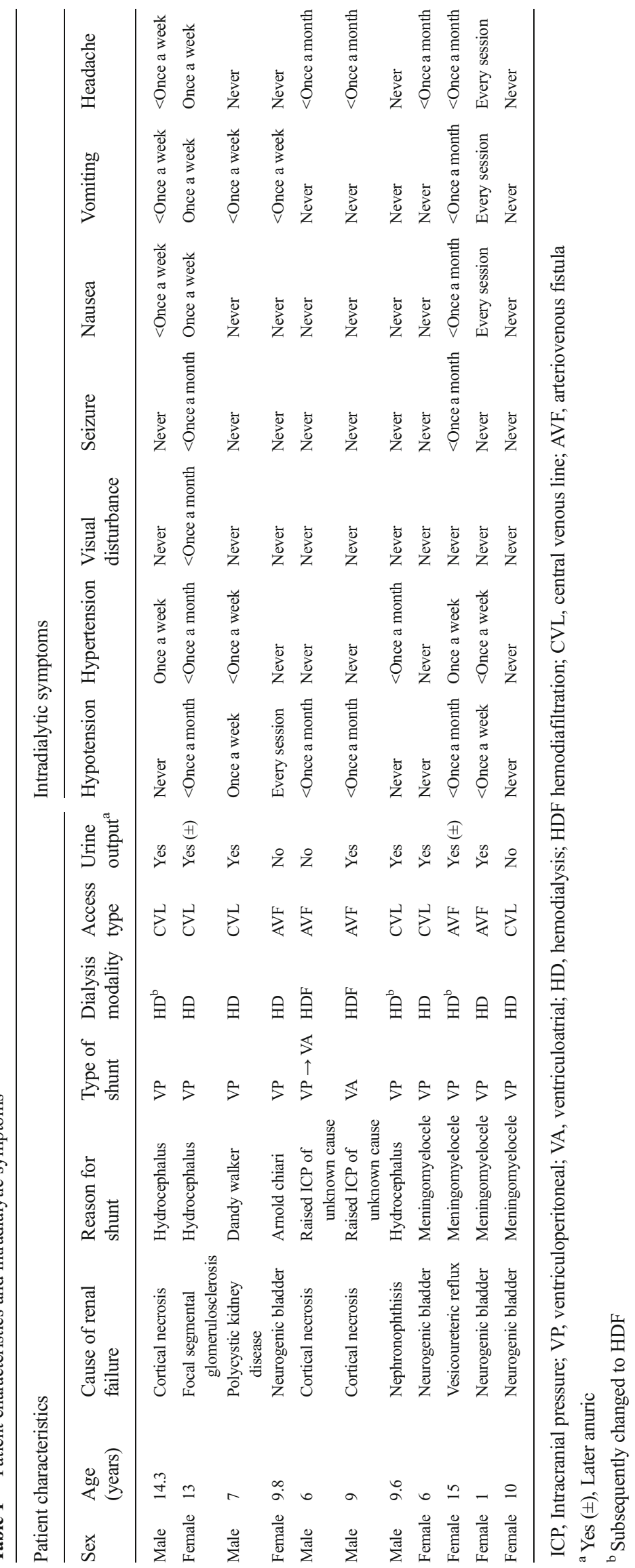


Table 2 Symptoms of hemodynamic instability reported by the children with a ventriculoperitoneal shunt (VPS) on hemodialysis $(n=11)$

\begin{tabular}{|c|c|c|c|c|}
\hline \multirow[t]{2}{*}{ Symptoms experienced } & \multicolumn{4}{|c|}{ Number of children experiencing symptoms and frquency of symptoms ${ }^{\mathrm{a}}$} \\
\hline & $\begin{array}{l}\text { Every session/at least } \\
\text { weekly }\end{array}$ & $<$ Once a week & $<$ Once a month/infrequent & Never \\
\hline Hypotension & 2 & 1 & 4 & 4 \\
\hline Hypertension & 2 & 2 & 2 & 5 \\
\hline Visual disturbances & 0 & 0 & 1 & 10 \\
\hline Nausea & 2 & 1 & 1 & 7 \\
\hline Vomiting & 2 & 3 & 1 & 5 \\
\hline Headache & 2 & 1 & 4 & 4 \\
\hline Seizures & 0 & 0 & 2 & 9 \\
\hline
\end{tabular}

${ }^{\text {a }}$ Respondents were asked to rate symptom frequency as 'every session'/'once a week', 'less than once a week', 'less than once a month' or 'never'

\section{Discussion}

In this 10 -year survey across 15 pediatric dialysis units in Europe we have shown that HD in children with a VPS is safe, with no VPS infection or meningitis even in those with dialysis catheter infections. However, our survey suggests that HD in the presence of a VPS is associated with frequent symptoms of hemodynamic instability and seizures, making the dialysis therapy more complicated to manage and difficult for the child to tolerate.

There is little known about dialysis in the presence of a VPS, either in adults or children. The number of children with VPS requiring chronic dialysis is very low, with 18 cases of PD (prevalence $0.6 \%$ ) [5] and 11 cases of HD (prevalence $1.33 \%$ ) in children with a VPS in our survey. The decision to start HD a child with a VPS or a VA shunt is significant and usually subject to medical discussion regarding the selection of treatment modality. In our survey each center had carefully searched through their HD databases in order to correctly identify all patients with a VPS. According to a recent survey from the IPPDN, PD in the presence of a VPS is safe, with no increase in the risk of either VPS infections or peritonitis [5]. Our survey is the first report on the safety and feasibility of HD in children with a VPS based on a multicenter survey.

There are concerns about performing dialysis in patients with a VPS due to the risk of cross-infections between the dialysis catheter and the VPS or a risk of ascending infection from the peritoneum. However, a study performed 25 years ago demonstrated that PD with a VPS is feasible [6]. In a study of peritonitis rates in children with spina bifida on PD, four episodes of peritonitis were reported in three children with a VPS, but none of the children developed concomitant VPS infection [3]. The IPPDN Registry reports 20 episodes of peritonitis in 11 of 18 children on PD with a VPS, giving a peritonitis rate of 1 in 19.6 patient-months [5]. The Registry reported no ascending infections associated with peritonitis although there were 20 peritonitis episodes recorded during the study period. One child developed a shunt infection, but this was in the absence of peritonitis. Four children were transferred to HD, but the change in dialysis modality was not due to a VPS-related malfunction or infection.

There is a single report of VPS-associated sepsis in a patient on HD. This was a case of coagulase-negative Staphylococcus VPS infection in an adult on HD dialyzed via an AVF [4]. Diagnosis was confirmed by lumbar puncture and the culture of free fluid sampled in the peritoneum; all blood cultures remained sterile, although the patient had spikes of fever during dialysis. The patient had been on HD for 5 years with the VPS in situ before the start of HD and was successfully treated and continued on HD. There is a second report of an iatrogenic VPS infection following insertion of an HD catheter which resulted in traumatic damage to the shunt with cerebrospinal fluid (CSF) leakage [7]. Our survey identified three HD line infections, but none resulted in ascending infection of the shunt.

The majority of children in our survey had symptoms of hemodynamic instability on dialysis. Three children (27\%) were switched from HD to HDF in an attempt to achieve better control of their fluid status. This change in dialysis modality was reported to have reduced the frequency of intradialytic symptoms in two children, but these were not children who experienced frequent symptoms. Intradialytic hypotension is a common complication of dialysis, and reported rates in adults range from 17 to $31 \%[8,9]$; there are no comparable data in children [10]. We found a significantly lower prevalence of hypotensive complications in children without VP shunts. Acute reductions in myocardial blood flow (myocardial stunning) during conventional HD which correlate with intradialytic blood changes and ultrafiltration volume have been demonstrated in children [11]. The typical management of intradialytic hypotension is to either reduce or stop ultrafiltration, administer a bolus of saline or discontinue the session prematurely [10]. These responses had little effect in controlling the symptoms of the affected patients in our survey. 
Despite the frequency of symptoms, no child was switched to PD. There are no reports of hemodynamic instability in children with a VPS receiving PD to allow for comparison with those receiving HD or HDF.

Hemodiafiltration has been shown to reduce intradialytic hypotension. In a randomized controlled trial of adults on HD, HDF and hemofiltration, intradialytic symptomatic hypotension was recorded in $7.5 \%$ of sessions, with significantly fewer episodes among patients on HDF compared to those on HD [12]. Fewer symptoms of intradialytic hypotension in patients treated with HDF compared with HD have also been reported [13]. Daily 3-h HDF sessions six times per week have been shown to be more effacious than daily 4-h HDF sessions three times a week, with a reduction in blood pressure, improvement in left ventricular size and function, an enhanced feeling of general well-being and no post-dialysis recovery time $[14,15]$. No center participating in our survey reported increasing the hours of daily dialysis to alleviate the symptoms of hemodynamic instability.

The use of prophylactic mannitol, an osmotic agent, has been reported to half the odds of intradialytic symptoms and increase tolerance to ultrafiltration [16]. Mannitol was used in two of the children in our survey, but it did not improve symptoms. The secretion of CSF is variable, with adults producing as much as $750 \mathrm{ml}$ a day [17]. The child in our study who had the VPS externalized demonstrated variation in drainage: during HD the shunt drained approximately $300 \mathrm{ml}$ over $4 \mathrm{~h}$, whereas the same volume was drained over $24 \mathrm{~h}$ when the child was off dialysis (personal observation). Fluid shifts during dialysis, in particular the very rapid movement of CSF, may account for the hemodynamic instability seen in some children on dialysis.

Regardless of dialysis modality, the VPS is susceptible to a range of complications, including mechanical blockage, valve failure, disconnection and infection [18]. The authors of a longitudinal study of VPS in adults and children reported surgical shunt complications in $35 \%$ of the patients over a mean follow-up period of 4.5 years. Children, particularly neonates, experienced the highest complication rates [19]: almost half of the children required a repeated surgical procedure by 5 years of follow-up. None of our patients developed a VPS-related surgical complication despite a comparable follow-up period. Encapsulating sclerosing peritonitis, which is most commonly associated with chronic PD [20,21], has also been reported in two children with VP shunts who were not on dialysis [22].

Our study has a number of limitations common to many multicenter retrospective surveys. Patients were identified by recall and underreporting is possible but unlikely as HD in the presence of a VPS is a significant management issue on a pediatric dialysis unit. A prospective audit across all European dialysis centers through Registry data would give a greater perspective on the prevalence, dialysis management and complications of children with a VPS on HD. Comparison with a cohort of children without VPS would give a clear idea if the reported hemodynamic instability was caused by the VPS or due to fluid shifts that are seen in chronic HD patients. We were unable to obtain data from children dialyzed during the same study period as our study cohort, and the significantly lower rate of hemodynamic complications in our current HD patients without VP shunts may reflect improved dialysis management.

In conclusion, our survey suggests that HD in children with a VPS is a safe procedure, with no episodes of VPS infection or meningitis even in those with CVL sepsis. However, an increased frequency of hemodynamic instability can make the dialysis therapy more complicated to manage and difficult for the child to tolerate. As shown in studies with adult HD patients, HDF may also decrease hemodynamic instability in patients with VPS.

Conflict of interest The authors declare no conflict of interest.

\section{References}

1. Kato N, Chin-Kanasaki M, Tanaka Y, Yusuda M, Yokomaku Y, Sakaguchi M, Isshiki K, Araki S, Ohta S, Uzu T (2011) Successful renal replacement therapy for a patient with severe haemophilia after surgical treatment of intercranial haemorrhage and hydrocephalus. Case Rep Nephrol 2011: 824709

2. Grunberg J, Rebori A, Verocay MC (2003) Peritoneal dialysis in children with spina bifida and ventriculoperitoneal shunt: one center's experience and review of the literature. Perit Dial Int 23:481486

3. Grunberg J, Verocay MC, Rebori A, Pouso J (2007) Comparison of chronic peritoneal dialysis outcomes in children with and without spina bifida. Pediatr Nephrol 22:573-577

4. Thet Y, Myint W, Hughes D, Crowe AV (2008) Ventriculoperitoneal shunt infection in a patient on hemodialysis. Hemodial Int 12:319-321

5. Dolan NM, Borzych-Duzalka D, Suarez A, Principi I, Hernadez O, Al-Akash S, Alconchar L, Breen C, Fischbach M, Flynn J, Pape L, Piantanida JJ, Printza N, Wong W, Zaritsky J, Schaefer F, Warady BA, White CT (2013) Ventriculoperitoneal shunts in children on peritoneal dialysis: a survey of the International Pediatric Peritoneal Dialysis Network. Pediatr Nephrol 28:315-319

6. Warady BA, Hellerstein S, Alon U (1990) Advisability of initiating chronic peritoneal dialysis in the presence of a ventriculoperitoneal shunt. Pediatr Nephrol 4:96

7. Wang H-Y, Lin W-Y (2010) An unusual iatrogenic cerebrospinal fluid leakage from ventriculoperitoneal shunt. Ann Nucl Med Sci 23:59-63

8. Sands J, Usvyat L, Sullivan T, Segal J, Zabetakis P, Kotanko P, Maddux F, Diaz-Buxo J (2014) Intradialytic hypotension: frequency, sources of variation and correlation with clinical outcome. Hemodial Int 18:415-422

9. Stefansson B, Brunelli S, Cabrera C, Rosenbaum D, Anum E, Ramakrishnan K, Jensen D, Stalhammar N (2014) Intradialytic hypotension and risk of cardiovascular disease. Clin J Am Soc Nephrol 9:2124-2132

10. Hayes W, Hothi D (2011) Intradialytic hypotension. Pediatr Nephrol 26:867-879 
11. Hothi D, Rees L, Marek J, Burton J, McIntyre C (2009) Pediatric myocardial stunning underscores the cardiac toxicity of conventional hemodialysis treatments. Clin J Am Soc Nephrol 4:790-797

12. Locatelli F, Altieri P, Andrulli S, Bolasco P, Sau G, Pedrini L, Basile C, David S, Feriani M, Montagna G, Raffaele di Iorio B, Memoli B, Cravero R, Battaglia G, Zocalli C (2010) Hemofiltration and haemodiafiltration reduce intradialytic hypotension in ESRD. J Am Soc Nephrol 21:1798-1807

13. Caplin B, Alston H, Davenport A (2013) Does online haemodiafiltration reduce intra-dialytic patient symptoms? Nephron Clin Pract 124:184-190

14. Fischbach M, Terzic J, Laugel V, Dheu C, Menouer S, Helms P, Livolsi A (2004) Daily on-line haemodiafiltration: a pilot trial in children. Nephrol Dial Transplant 19:2360-2367

15. Fischbach M, Terzic J, Menouer S, Dheu C, Seuge L, Zalosczic A (2010) Daily online haemodiafiltration promotes catch-up growth in children on chronic dialysis. Pediatr Nephrol 25:867-873

16. Hothi D, Harvey E, Goia CM, Geary D (2009) The value of sequential dialysis, mannitol and midodrine in managing children prone to dialysis failure. Pediatr Nephrol 24:1587-1591
17. Greenberg MS (2010) Handbook of neurosurgery, 7th edn. Thieme, Stuttgart

18. Stein S, Guo W (2007) A mathematical model of survival in a newly inserted ventricular shunt. J Neurosurg Pediatr 107:448-454

19. Wu Y, Green N, Wrensch M, Zhao S, Gupta N (2007) Ventriculoperitoneal shunt complications in California: 1990 to 2000. Neurosurgery 61:557-562

20. Shroff R, Stefanidis CJ, Askiti V, Edefonti A, Testa S, Ekim M, Kavaz A, Ariceta G, Bakkaloglu S, Fischbach M, Klaus G, Zurowska A, Holtta T, Jankauskiene A, Vondrak K, Vande Walle J, Schmitt CP, Watson AR, on behalf of the European Pediatric Dialysis Working Group (2013) Encapsulating peritonitis sclerosis in children on chronic PD: a survey from the European Pediatric Dialysis Working Group. Nephrol Dial Transplant 28:1908-1914

21. Stefanidis CJ, Shroff R (2014) Encapsulating peritoneal sclerosis in children. Pediatr Nephrol 29:2093-2103

22. Sigaroudinia MO, Baillie C, Ahmed S, Mallucci C (2008) Sclerosing encapsulating peritonitis - a rare complication of ventriculoperitoneal shunts. J Pediatr Surg 43:E31-E33 